served change in course at 0.06 alcohol mole fraction appearing as a shoulder at $10^{\circ} \mathrm{C}$. which progressively developed with increasing temperature to furnish a small but definite peak at $30^{\circ} \mathrm{C}$.

Elsewhere ${ }^{3}$, the increase of the solvent water partial molar volume has been interpreted as arising from the orientation of hydration shell water adjacent to the ethyl group of the alcohol. It is now proposed that the alcohol partial molar volume anomaly at 0.06 alcohol mole fraction derives directly from, and provides direct evidence for, the existence of a three-dimensionally hydrogen-bonded liquid hydrate of composition $\mathrm{C}_{2} \mathrm{H}_{5} \mathrm{OH} \cdot 17 \mathrm{H}_{2} \mathrm{O}$. Such a liquid hydrate would involve 28 -fold co-ordination of the alcohol molecule by water with the alcoholic-OH group hydrogen-bonded to the water cage, the solution structure being likened in respect to short-range order to a broken-down structure II gas-hydrate ${ }^{4}$ of formula $M \cdot 17 \mathrm{H}_{2} \mathrm{O}$.

This interpretation provides a direct structural basis of the older hydrate theory of aqueous solution as developed by Pickering 5 in 1893 , provided that the aqueous solutes are limited to non-electrolytes and that the meaning of a molecule of hydrate in solution is understood in the general erystallographic sense as the composition of the solution repeat unit, bearing in mind that the solution repeat unit composition does not define the hydration number of the solute but is structurally and geometrically related to it. The solid gas hydrates ${ }^{4}$ of dimethyl ether, ethylene oxide and of acetone, lead one to expect that the molar volume anomaly presented by aqueous ethyl alcohol will not be limited to ethyl alcohol alone, but will be generally exhibited in dilute aqueous solutions of many of the smaller, soluble, organic non-electrolytes.

$$
\text { D. N. Guew }
$$

Exploratory Research Laboratory,

Dow Chemical of Canada, Ltd.,

Sarnia, Ontario.

1 Franks, F., and Johnson, H. H., Trans. Farad. Soc., 58, 656 (1962). ${ }^{2}$ Mitchell, A. G., and Wynne-Jones, W. F. K., Disc. Farad. Soc. No. 15,161 (1953)

' Glew, D. N., J. Phys. Chem., 66, 605 (1962).

- Stackelberg, M. von, and Müller, H. R., Z. Elektrochem., 58, 25 (1954).

'Pickering, S. U., Trans. Chem. Soc. 63, 141 (1893).

\title{
Fluorescence of Quinine in an Alkaline Medium and in Absolute Ethanol
}

Papers dealing with fluorescence of quinine only give the characteristics of this compound in an acid medium $^{1-4}$. Some publications suggest that quinine does not fluoresce at all in an alkaline medium ${ }^{5,6}$.

In our experiments with quinine we discovered some fluorescent characteristics of quinine in an alkaline medium and in absolute ethanol which so far as we know have not been mentioned previously (Table 1). The maxima in an acid medium are given for comparison. The ratio of intensity of the fluores-

Table 1. Exchtation and Fluorescence Maxima of QUinine

Figures between brackets indicate that there is no distinct maximum but only a shoulder
buts between brackets indicate that there

Medium

$0 \cdot 1 \mathrm{~N}$ sulphuric acid

Absolute ethanol

$0.01 \mathrm{~N}$ sodium

hydroxide

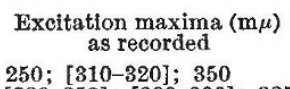

250 ; [310-320]; 350

[230-250]; [280-290]; 327

[230-250]; [280-290]; 327
Fluorescence maximum 442 350 365
Table 2. ABSORPTION OF QUININE $(10 \mu \mathrm{g} / \mathrm{ml}$.) IN DIFFHRENT MEDIA THE OPTIOAL DENSITY IS GIVEN BETWEEN BRACKETS

Medium Wave-lengths of maximum absorption $(\mathrm{m} \mu)$ $0.1 \mathrm{~N}$ sulphuric acid Absolute ethanol 01 N sodium 250 [0.900];316 [0.125]; 348 [0.165] $234[0.900] ; 316[0.125] ; 348[0.165] ; 330.80 .160]$ $231[0.940] ; 282[0 \cdot 115]$; $327[0 \cdot 145]$

cence in the media mentioned is approximately $1: 10: 60$ for alkali, alcohol and acid respectively.

We used a Farrand spectrophotofluorometer equipped with a $1 P 28$ photomultiplier tube, a penand-ink recorder (Micrograph from Kipp, Delft) and a $10 \times 20 \times 50 \mathrm{~mm}$ Farrand quartz cuvette. The entrance slits were $2 \mathrm{~mm}$, the exit slits $0.5 \mathrm{~mm}$ for both monochromators.

The differences in excitation maxima might be due to artefacts; however, the ultra-violet absorption spectra of quinine $(10 \mu \mathrm{g} / \mathrm{ml}$.) in the foregoing media showed that the wave-lengths of maximum absorption (Table 2) were in good agreement with the excitation maxima in Table 1. Such an agreement between excitation and absorption maxima has already been demonstrated for quinine in an acid medium ${ }^{3}$.

The fact that the fluorescent characteristics of quinine in different media are quite distinct may be used for a better identification of this compound in qualitative analysis.

\section{H. A. LINNEWIEI}

B. J. VISSER

Pharmaco-Therapeutic Laboratory, University of Amsterdam.

${ }^{2}$ Udenfriend, S., Duggan, D. E., Vasta, B. M., and Brodie, B. B., J. Pharmacol. Exp. Therap., 120, 26 (1957)

${ }^{2}$ Sprince, H., and Rowley, G. R., Science, 125, 25 (1957).

${ }^{3}$ De Francesco, F., Ann. Chim. (Rome), 48, 390 (1958).

4 Melhuish, W. H., J. Phys. Chem., 64, 762 (1960).

Kavanagh, F., and Goodwin, R. H., Arch. Biochem., 20, 315 (1949).

- Knox, W. E., J. Biol. Chem., 163, 699 (1946).

\section{Influence of Deoxyribonucleic Acid on the Intermolecular Structure of Water}

SEVERAL years ago Jacobson ${ }^{1}$ directed attention to the fact that the helical structure of deoxyribonucleic acid (DNA) proposed by Watson and Crick ${ }^{2}$ showed a remarkable relationship to the intermolecular structure of liquid water, which has been postulated to be quasi-crystalline and only slightly different from ice ${ }^{3}$. Jacobson proposed that when DNA is dissolved in water, an increased ordering of the water structure results and further suggested this increased ordering of the intermolecular water structure accounts for the high viscosity and dielectric constant of DNA solutions. In later work Jacobson et al. ${ }^{4}$ studied the proton resonance spectra of DNA solutions and inter. preted the results of their experiments as offering further support of the above-mentioned proposals. Jacobson ${ }^{5}$ then made a detailed study of the dielectric constants of DNA solutions and interpreted the results as further evidence of stabilization of the normal water structure by the formation of hydration shells with diameters at least one-quarter the length of the dissolved macromolecules; he also cited certain $\mathrm{X}$-ray studies ${ }^{6}$ for further support of his hypothesis. The sodium salt of deoxyribonucleic acid (sodium thymonucleate) was used in all Jacobson's experimental work.

The work recorded here was undertaken with the view of obtaining infra-red data on the nature of the 